\title{
Embedded sensing: integrating sensors in 3-D printed structures
}

\author{
Alexander Dijkshoorn, Patrick Werkman, Marcel Welleweerd, Gerjan Wolterink, Bram Eijking, \\ John Delamare, Remco Sanders, and Gijs J. M. Krijnen \\ Robotics and Mechatronics group, MIRA institute, University of Twente, Enschede, The Netherlands
}

Correspondence: Gijs J. M. Krijnen (gijs.krijnen@utwente.nl)

Received: 26 September 2017 - Revised: 21 December 2017 - Accepted: 31 December 201 - Published: 23 March 2018

\begin{abstract}
Current additive manufacturing allows for the implementation of electrically interrogated 3-D printed sensors. In this contribution various technologies, sensing principles and applications are discussed. We will give both an overview of some of the sensors presented in literature as well as some of our own recent work on 3-D printed sensors. The 3-D printing methods discussed include fused deposition modelling (FDM), using multi-material printing and poly-jetting. Materials discussed are mainly thermoplastics and include thermoplastic polyurethane (TPU), both un-doped as well as doped with carbon black, polylactic acid (PLA) and conductive inks. The sensors discussed are based on biopotential sensing, capacitive sensing and resistive sensing with applications in surface electromyography (sEMG) and mechanical and tactile sensing. As these sensors are based on plastics they are in general flexible and therefore open new possibilities for sensing in soft structures, e.g. as used in soft robotics. At the same time they show many of the characteristics of plastics like hysteresis, drift and non-linearity. We will argue that 3-D printing of embedded sensors opens up exciting new possibilities but also that these sensors require us to rethink how to exploit non-ideal sensors.
\end{abstract}

\section{Introduction}

Additive manufacturing (AM), more colloquially known as 3-D printing, is a fabrication technology in which parts are built layer-by-layer from a digital description using one of a variety of methods to deposit and solidify specific materials. The technology has been around for more than 3 decades but recently the number of materials that can be printed, the resolution and speed with which this can be done and the ability to combine more than one material in multi-material prints have been improved significantly. On the other hand the costs of printing and materials have steadily declined.

AM has been used traditionally to make non-functional structures for rapid prototyping purposes. However, recent developments in multi-material 3-D printing have started to trigger research into printing of functional structures (Leigh et al., 2012). For customised structures that already are made by AM, e.g. robotics and prosthetics, embedding 3-D printed sensors seems a promising next step to increase functionality. The development of this technology will allow application of sensors, independent of commercial-off-the-shelve (COTS) components, since customisation in performance, packaging and interfacing, as well as integration in/with complex shapes, will lead to unprecedented possibilities. Where the performance of such sensors will have the burden of proving their viability relative to other well-established sensor technologies like micro-electromechanical systems (MEMS) and precision engineering (Krijnen and Sanders, 2016), it is clear that developments in e.g. soft robotics, prosthetics and orthotics and other developments based on "soft materials" require sensors largely different from current COTS sensors. At the same time fully integrated manufacturing may come at reduced costs since no assembly will be required.

Evidently, AM for embedded sensing will form an additional technology with respect to e.g. photolithographybased methods (micro-electromechanical systems (MEMS) and nanotechnology), computer numerical control (CNC) machines and moulding approaches. However, it provides a unique set of characteristics that sets it apart from these other methods; see Table 1. Therefore it is more likely that AM will create its own application field, probably complementing 
Table 1. Some key features of various fabrication technologies n.a. stands for "not applicable".

\begin{tabular}{lllll}
\hline Technology & Photolithography & 3-D printing & CNC & Moulding \\
\hline Dimensionality & 2.5-D (stratified) & 3-D & 3-D, limited & 3-D, limited \\
Fabrication & Batchwise & Piecewise & Piecewise & Batchwise \\
Materials & Inorganic & Organic & Inorganic & Organic \\
& Mostly stiff & Stiff \& flexible & Stiff & Stiff \& flexible \\
& Linear & Creep, hysteresis & Linear & Creep, hysteresis \\
Resolution & $\mathrm{nm}-\mu \mathrm{m}$ & $\mu \mathrm{m}-\mathrm{mm}$ & $0.1 \mu \mathrm{m}-\mathrm{mm}$ & $\mu \mathrm{m}-\mathrm{mm}$ \\
\$/piece & Low & High & High & Low \\
\$/batch & High & n.a. & n.a. & High \\
Customisation & None & High & High & None \\
Packaging & Expensive & Inclusive & Additional & Additional \\
Lead time & Long & Short & Short & Long \\
\hline
\end{tabular}

other fabrication methods, rather than competing with these established methods.

Clearly, embedded sensing and actuation cannot do without proper electronic underpinning. However, despite the wide interest in (3-D) printed electronics, we do not consider such integration in this paper since we believe that these developments will become available only further into the future and that also interesting embedded sensor work can be done without embedded electronics. Nevertheless the combination of electronic components with 3-D printed structures through manual or (semi-)automated assembly has already been demonstrated (Shemelya et al., 2015a) and will allow tight integration with embedded transduction. In this paper we only consider the combination of dielectric with electrically conductive materials, which is sufficient to make a range of embedded sensors.

\subsection{Current technology for embedded sensors}

Currently there are at least three methods to combine dielectric with conductive parts in order to make sensors and actuators:

1. hybrid approaches, i.e. combining printed parts with non-AM fabricated structures, e.g. regular wiring, printed circuit boards or entire sensors;

2. conductor infusion, i.e. printing channels in otherwise non-conductive materials by arbitrary AM methods with subsequent infusion of conductive inks (Wu et al., 2015); and

3. multi-material printing, i.e. combining the use of conductive and non-conductive filaments (Leigh et al., 2012), predominantly by fused deposition modelling (FDM).

Method (1) allows for a straightforward combination of parts, largely comparable to classical assembly. The disadvantage is that the potential of AM is only materialised in a very limited way. The advantage of (3) is the promise of straightfor- ward integration, the disadvantages being the limited availability of suitable material combinations, anisotropic conduction properties and relatively high $\left(10^{-4}\right.$ to $\left.1 \Omega \mathrm{m}\right)$ resistivities. Method (2) does not have these disadvantages but homogeneously filling channels with conductive inks is challenging, especially when filling needs to be obtained by pressure-driven flows and through a diverging network of channels. With respect to electrical interfacing, both methods (2) and (3) can show considerable variations in contact resistances, subject to mechanical loading, temperature and humidity changes, etc.

\subsubsection{Hybrid approaches}

Combining 3-D printing with (automated) assembly of e.g. wiring and electronic parts can offer substantial benefits. For example conductors can be made of the material of choice for the respective application, e.g. copper wiring to allow for the largest possible current densities and, hence, minimum losses by resistive heating. However, the method is also cumbersome in the sense that it does not allow to have full freeform placement of conductors and requires extensive mechatronic equipment to combine wire routing with $3-\mathrm{D}$ printing. An example of this approach is the stop-and-go method developed at the Keck Center at the University of Texas in El Paso (MacDonald et al., 2014).

\subsubsection{Infused conductors}

Infusion of conductive materials in dielectric prints is possible by using dissolvable support material to form (networks of) channels. In principle this method allows for complicated electrical wiring since the channels are formed in full freeform fabrication. Nevertheless the dissolving of the support material can be tricky for complicated and narrow channels, and the actual infusion even more so.

Inks and paints can play an important role in additive manufacturing and are mostly found as particulate suspensions: 
1. suspensions in which the carrier remains liquid after infusion (Ota et al., 2016);

2. suspensions in which the carrier solidifies after infusion (Czyzewski et al., 2009; Vatani et al., 2015), e.g. by light curing (Vatani et al., 2015), resin developing (Muth et al., 2014) or evaporation of solvents (Pella); and

3. suspensions in which the carrier evaporates after infusion (Ko et al., 2010; Wu et al., 2015; Harada et al., 2014).

The first two have the advantage that void spaces can easily be filled on the condition that the suspension remains mono-disperse. When using a suspension in which the solvent evaporates, it remains uncertain as to where the particles will settle and voids will be present. This problem can partly be solved by repeatedly filling the channels after the solvent has evaporated (Wu et al., 2015).

Inks and paints have a wide range of possible resistivities. Not only is there a diverse array of conductive particles available such as silver, gold and several allotropes of carbon (Lopes et al., 2014; Liu et al., 2003; Boland et al., 2014; Song et al., 2017; Zin et al., 2015; Zhao et al., 2013), but also the ratio of filler content to the entire solution can be altered to get the desired conductivity (Zhao et al., 2013; Ota et al., 2016). Furthermore, to high surface-area-to-volume ratios, the melting point of materials can be reduced, promoting sintering between the particles under mild conditions, leading to resistivities close to the bulk resistivity of the material (Habas et al., 2010; Lopes et al., 2014). A condition for using this technique is that the 3-D print can sustain the elevated temperatures needed for this.

Particulate tracks generally have high gauge factors. Next to the change in geometry of the conductive track, changes in resistivity can be observed. This can be ascribed to numerous connections and disconnections between neighbouring nanoparticles or microparticles. While this effect makes for higher gauge factors, it is also a cause for hysteresis effects and drift in the absolute value of the strain gauges. Drift is said to be the result of permanent cracks in the conductive track on the microscale (Borghetti et al., 2016; Song et al., 2017; Boland et al., 2014).

\subsubsection{Multi-material co-printed conductors}

From a viewpoint of design freedom and straightforward fabrication, co-printing conductors, i.e. conductive materials printed in the same print cycles as the dielectric materials, is the most desirable technology. However, at the current state of the art it comes at a price; i.e. the conductivities are still about $10^{3}-10^{5}$ times smaller than copper. Moreover printed conductive material shows significant anisotropic conduction, which especially in the $z$ direction is relatively poor. Additionally, given the multi-material printing nature of this

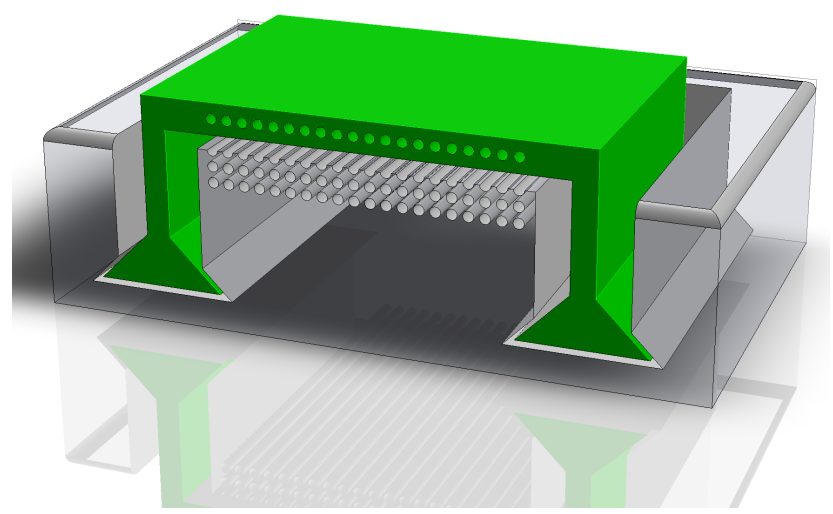

Figure 1. Conceptual design of a 2DOF capacitive force sensor. The green part is flexible. Holes represent position of conductive wire (Shemelya et al., 2015b).

method not all material combinations are suitable since some may require incompatible printing conditions, e.g. bed temperature. Currently the most advanced multi-material methods, i.e. those based on poly-jetting of materials, do not yet provide any possibility to print conductive parts, a situation that may, however, change in the near future.

\subsection{Sensing principles}

As with other technologies for sensor fabrication, depending on materials properties and technological characteristics, various sensing principles may be applicable - some requiring only an appropriate geometry, others needing specific material properties as well.

\subsubsection{Strain gauges and piezoresistive sensing}

Strain gauge sensors can be straightforwardly printed since virtually each conductor shows a dependence on its mechanical loading, through geometry changes and/or by piezoresistive effects. The use of 3-D printing allows conductive traces to be positioned ideally for the expected loading, e.g. as shown in Fig. 14, for a clamped-clamped beam loaded from the top. However, the variation in contact resistance between the printed conductor and the external leads that may occur when parts are deformed poses some difficulties. Good results to depress this effect may be obtained by four-wire measurements. In contrast to metallic strain gauges which can have high linearity, printed strain gauges seem prone to creep, hysteresis and non-linear response, partly resulting from the host material properties, partly from the conductive material itself.

\subsubsection{Capacitive sensing}

Since 3-D printed conducting materials cannot easily be printed in thin layers, an interesting question is how well 


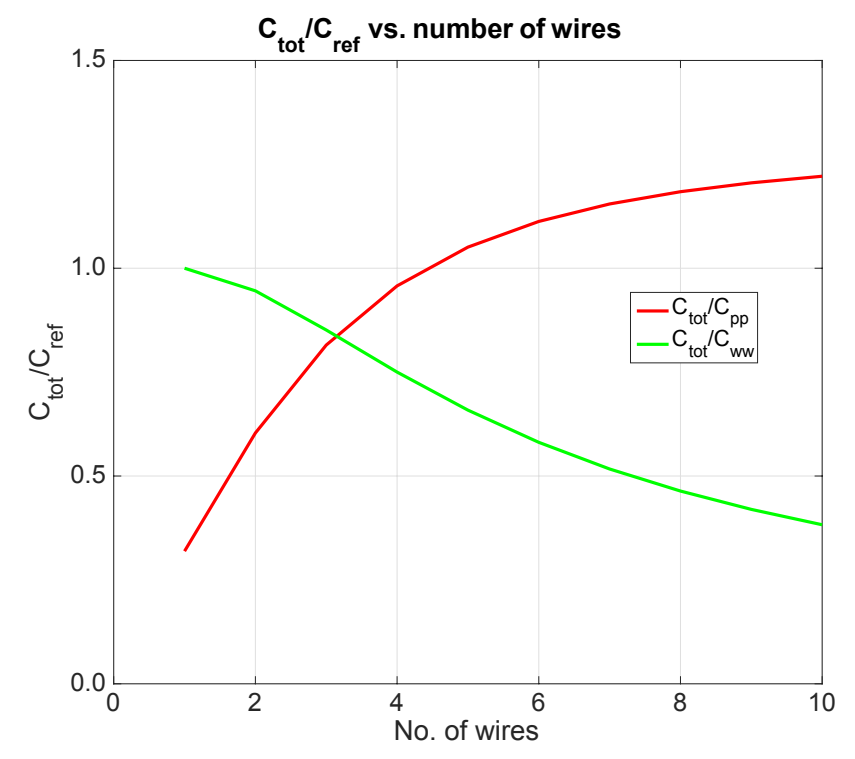

Figure 2. Relative capacitance versus the number of wires, where 10 represents a completely closed layer (Shemelya et al., 2015b).

capacitive sensors can be obtained by a mere collection of wires (see Fig. 1 for the design of a 2 degrees of freedom (DOF) force sensor), e.g. to be used in combination with an infusion process or a hybrid approach. See for example the work by Shemelya et al. (2015b), on copper wire and copper mesh embedding in fusion deposition modelling (FDM) printing, demonstrating capacitive touch, salinity and object sensing.

We modelled the capacitance as a function of density of wires (Shemelya et al., 2015a). It shows that at $50 \%$ coverage of the conductive plane the capacitance is close to the parallel plate approximation; see Fig. 2. We also modelled and experimentally investigated coplanar sensors (Fig. 3), consisting of interdigitated fingers with dielectric loading, offering possibilities for combining printed-circuit-boardbased (PCB-based) read-out with single material printed transducer structures (Fig. 6); see section below.

\subsubsection{Piezoelectric sensing}

3-D printed piezoelectric materials are under active research. A complicating factor for these type of materials is that they generally require some form of poling in order to manifest the piezoelectric microscopic properties on macro-scale structures. A material which seems to have interesting properties, both from a piezoelectric as well as an AM perspective, is polyvinylidene fluoride (PVDF) (Rajala et al., 2016). In Kirkpatrick et al. (2016) PVDF was poled during the printing process itself, yielding $d_{33}$ values of up to 0.36 (13) $\mathrm{pCN}^{-1}$.

\subsubsection{Magnetic sensing}

Magnetic sensing can be obtained in various ways, each requiring different structures and materials. In Leigh et al. (2014) layers of magnetite are printed in order to enable a Hall effect sensor to measure the rotation of a magnetised impeller in order to determine flow velocities. The work stresses the suitability of the technology to provide replacements for obsolete parts and shows better than original performance for the specific case studied.

In our own work we have attempted to use the pseudo-Hall effect to measure the rotation acceleration of a biomimeticvestibular-system-inspired sensor (van Tiem et al., 2015). The combination of a magnetic field and ion-loaded fluid flow produces a flow-dependent voltage difference. Unfortunately, in our sensors this signal was deeply covered in the electrochemically induced signals, requiring more research.

\subsubsection{Electrochemical sensing}

Where printed structures contain channels with electrolytes, flow and species sensing may be obtained through electrochemical sensing (van Tiem et al., 2015). The effect is based on the formation of space charge distributions (e.g. double layers) driven by electrochemical potentials and the subsequent disturbance of these space charges by fluid movement. Although the effects may be rather complex the actual sensing geometry may be as simple as to consist of only two or three electrodes in a fluid duct. However, dynamic properties may depend critically and intimately on the actual electrolyte constituents and concentrations, and additional complications may arise from pollution of the fluids by the printed material.

\section{Hybrid approaches}

Hybrid approaches can offer the best of both worlds: freeform fabrication and proven technology for specific parts, i.e. the read-out of a sensor. Although it presents limitations relative to multi-material printing it generally provides easy approaches and solutions for functional integration.

\section{Whisker-inspired tactile sensor}

We recently investigated possibilities for 3-D printing biomimetic whisker sensors for tactile purposes (Delamare et al., 2016). In these studies we set out for a hybrid integration approach: the whisker structure and its suspension are 3 D printed, whereas the read-out consists of a capacitive measurement of a coplanar capacitance, affected by a dielectric driven into the electric field of the capacitance. The implementation contains a PCB providing the required electrode structures. A schematic of the sensor is shown in Fig. 3.

Examples of the design and printed parts are shown in Fig. 4 . The rotation is enabled by a torsion beam with ellip- 


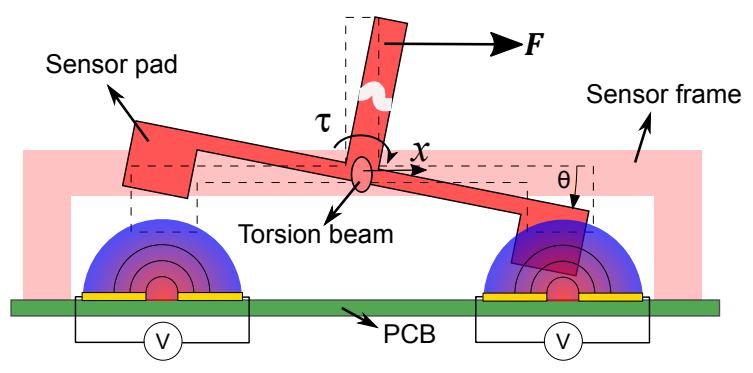

Figure 3. Coplanar capacitive sensing using interdigitated fingers with dielectric loading (Delamare et al., 2016).
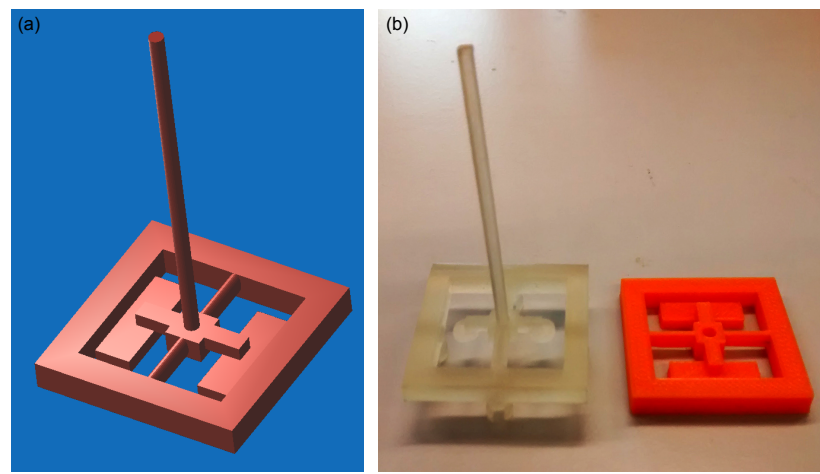

Figure 4. Panel (a) shows the 3-D CAD-design of whisker sensor in OpenScad (OpenScad). Panel (b) shows the printed whisker sensor (transparent, Veroclear) and base (red, PLA) (Delamare et al., 2016).

tical cross section, giving more compliance in the horizontal than in the vertical direction. An arbitrary force acting at an arbitrary position on the whisker will result in both a lateral force and a torque with subsequent rotation angle and lateral shift.

Figure 5 shows how the suspension behaves under mechanical loading. Overall the observed rotation angle is well in line with the predicted rotation angle. However, there is a significant difference between loading curves (open symbols) and unloading curves (solid symbols), reminiscent of mechanical hysteresis.

Although sensor structure and read-out were not integrated in this work we investigated the effect of the dielectric in the electrical field of the coplanar capacitor. Figure 6 shows the results of these measurements where we used an HP-4284A LCR meter to determine the capacitance and a linear translator to displace the dielectric. Two curves measured at $1 \mathrm{MHz}$ (red triangles and blue dots) clearly constitute a hysteresis curve. Also some odd behaviour can be seen at separation distances below $0.2 \mathrm{~mm}$ which we attribute to mechanical deformation of the dielectric pad and/or the PCB when mechanically loaded. We have shifted these curves $-0.2 \mathrm{~mm}$ to better reflect and compare them with the curve measured at $500 \mathrm{kHz}$ and the two calculated curves.

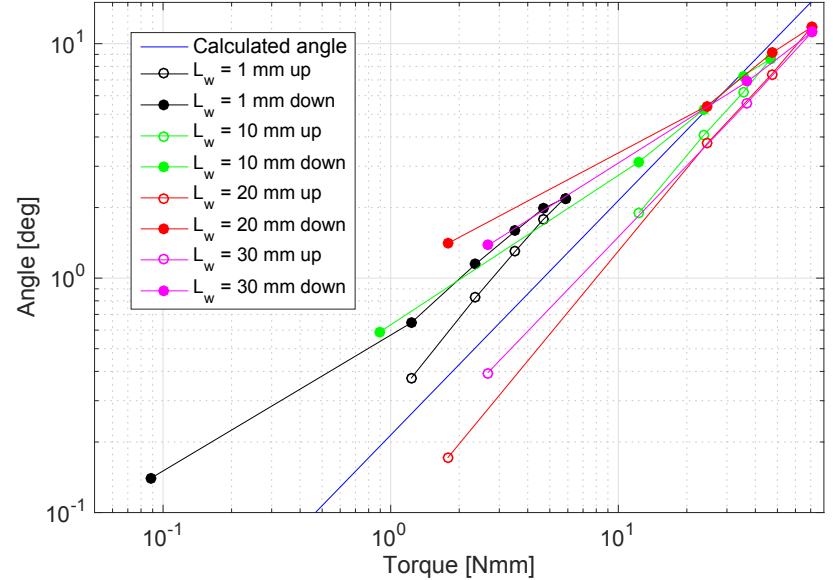

Figure 5. Rotation angle versus torque (Delamare et al., 2016).

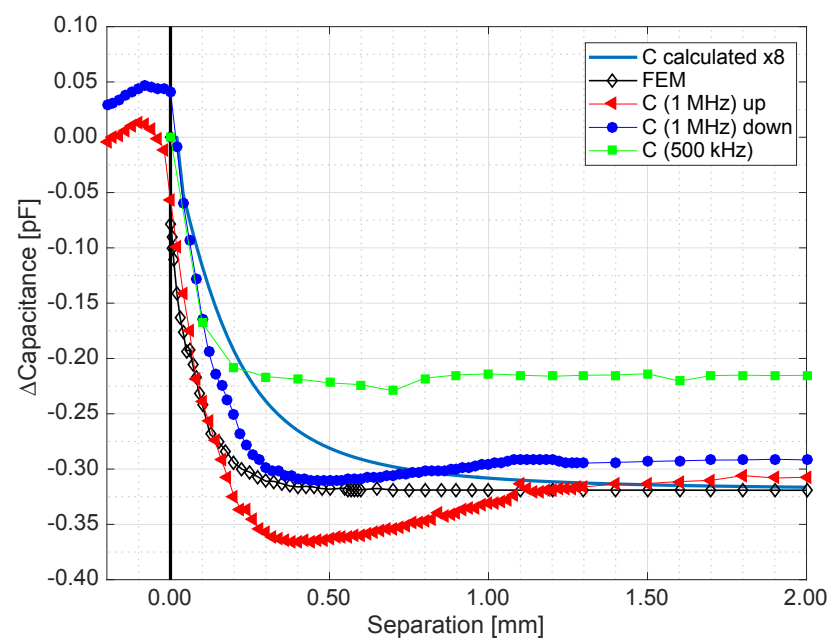

Figure 6. Coplanar capacitive sensing: capacitance versus distance of dielectric load (Delamare et al., 2016).

In the measurements we obtained a maximum change in capacitance of about $0.35 \mathrm{pF}$ relative to a base value of about $2.1 \mathrm{pF}$, i.e. $\Delta C / C \approx 0.17$. Comparable values are found in the FEM (finite element method) calculations but are underestimated by a factor of about 8 using conformal mapping calculations.

It is important to observe that both the mechanical as well as the capacitive measurements clearly show hysteresis behaviour, a feature observed with virtually all our 3-D printed sensors consisting of plastics.

\section{Infused conductors}

Making electrical conductors by filling channels has the benefit of having freedom of choice of the 3-D print process as long as it (a) allows for the use of soluble support material to make channels and (b) is impermeable to the fluids later to be 

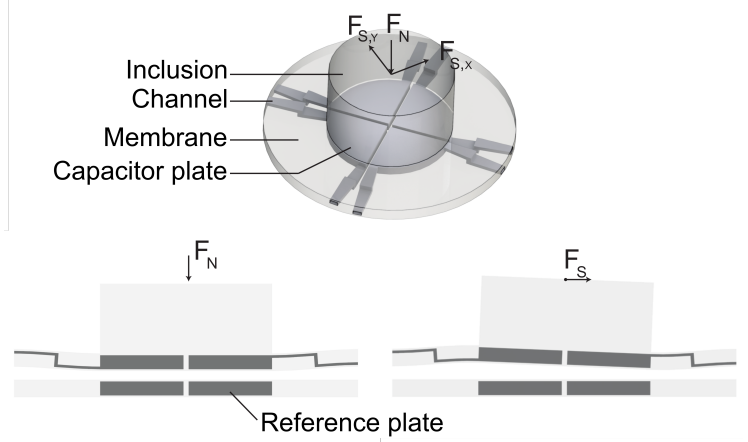

Figure 7. Design of a 3DOF force sensor with infused conductors.

infused into the channels. In principle wetting surfaces allow for filling by capillary forces but in practice the particle-laden fluids and glues have viscosities too high to obtain significant filling before drying. Further complications can stem from the need to fill complex networks and the need for a fine balance between the time to fill the channels and the time needed for solidification of the infused material ${ }^{1}$.

A nice example of infused conductors is the work of $\mathrm{Wu}$ et al. (2015). Using poly-jetting and FDM printing they proposed and demonstrated a range of passive electrical components. The combination of a few of these components was used to make an inductor-capacitor resonant tank with a resonance frequency at $0.53 \mathrm{GHz}$. They also demonstrated a 3-D printed smart cap with a comparable inductor-capacitor circuit, which shows a change in resonance frequency when exposed to decaying milk; i.e. a wireless measurement of milk held at $22^{\circ} \mathrm{C}$ showed significant changes in resonance frequency compared to milk held at $4{ }^{\circ} \mathrm{C}$ over a period of $36 \mathrm{~h}$.

\section{DOF force sensor}

In a recent research project (Welleweerd, 2017) we have investigated the possibilities of making a 3DOF force sensor by having an inclusion sitting on a membrane containing multiple strain gauges and capacitive sensors; see Fig. 7. The design freedom in AM allows channels to be made that follow the lines of maximum strain through the membrane, hence switching from top to bottom in the membrane in the current design (see also Fig. 14).

To investigate the quality of the infused conductors and study the mechanical and strain-gauge performance, a series of straight and crossover channels were made; see Fig. 8. Special care was taken to design appropriately shaped room for the header pins which otherwise may be subject to large variations in contact resistance. During 3-D printing the channels are temporarily filled by support material consisting of wax. To remove this wax afterwards the part is put in an oven at $60-65^{\circ} \mathrm{C}$ until the wax is melted and subsequently

\footnotetext{
${ }^{1}$ In principle fluidic conductors could be used as well but these tent to be expensive and require impermeable channel walls.
}

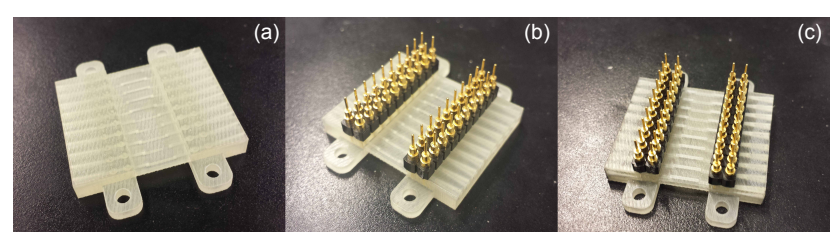

Figure 8. Assembly of a strain gauge sensor with straight channels. ((a)-(c)) The chip as received from Shapeways, the chip after insertion of headers and cleaning and the chip after infusion with graphite paint (Welleweerd, 2017).

cleaned by flushing the channels with olive oil. The channels were filled individually with graphite paint by a manually operated $1 \mathrm{~mL}$ syringe with sufficient but unknown pressure.

Mechanical loading tests were carried out on a test rig consisting of a voice coil actuator connected to a load cell via a rod sliding over an air bearing; see Fig. 9. Forces are applied by means of a plunger, connected to the load cell, whereas the resulting displacements are measured simultaneously. The system is set to a certain force after which the resistance of the 10 channels, the load cell and the displacement sensor are read out.

Mechanical response of the strain gauge to repetitive loading is shown in Fig. 10. Clearly the mechanical response shows (a) non-linearity, (b) hysteresis and (c) creep or drift as evidenced by the increasing deflection at near 0 load. The effects seem to be stronger when the structures are loaded from the bottom (left) than from the top (right). A non-linear beam model, taking into account beam stiffening and which is based on Senturia (2001), shows a reasonable correspondence in shape, excluding the hysteresis. However, the displacements at which beam stiffening seems to occur are relatively low, suggesting that effects other than beam stiffening (only) may be at play.

When looking at the electrical response, the mechanical response seems to be largely reflected though the creep seems to be more significant; see Fig. 11. Although far from perfect, the measurements clearly demonstrate the potential to use channels infused with conductive materials, both as conductors and strain gauges.

\section{Multi-material co-printed conductors}

There has been a steady increase in the availability of multimaterial printers (see e.g. Stratasys). Especially multi-nozzle FDM printers may process up to five materials (see ORD) from an ever-increasing range of specialty filaments. Some printers directly target co-printed conductive materials for printed electronics and embedded sensing (see Voxel8).

There are some good examples of co-printed conductive structures. Leigh et al. (2012) fabricated strain-sensing structures from carbon black doped polycaprolactone (PCL). Doping fractions of about $15 \%$ by weight were used to yield conductive filaments of about $0.1 \Omega \mathrm{m}$, where lower carbon 


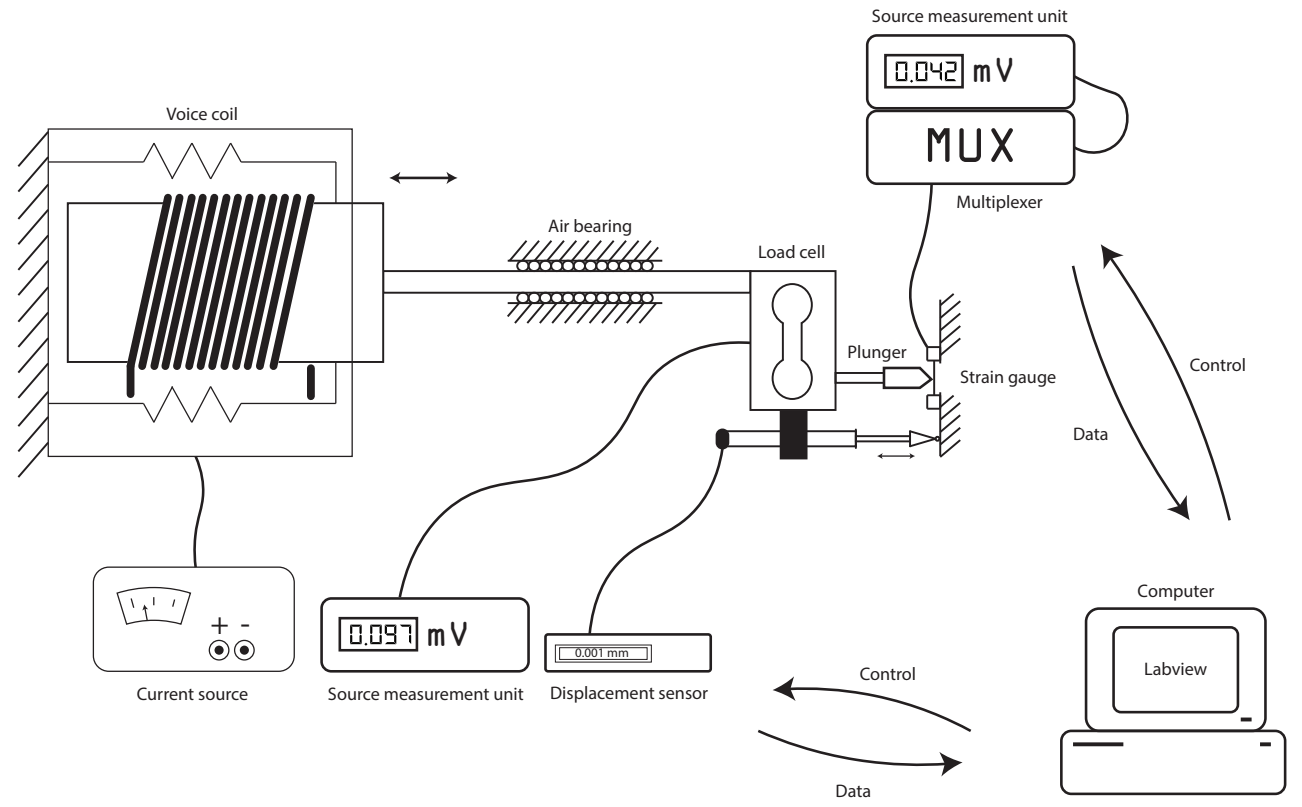

Figure 9. Measurement set-up to analyse the response of the strain gauges. A voice coil actuator is connected to a load cell via a rod which slides over an air bearing. On the other side of the load cell a plunger is connected which is pushed on the strain gauge. The system is set to a certain force after which the resistance of the 10 channels, the load cell and the displacement sensor are read out (Welleweerd, 2017).
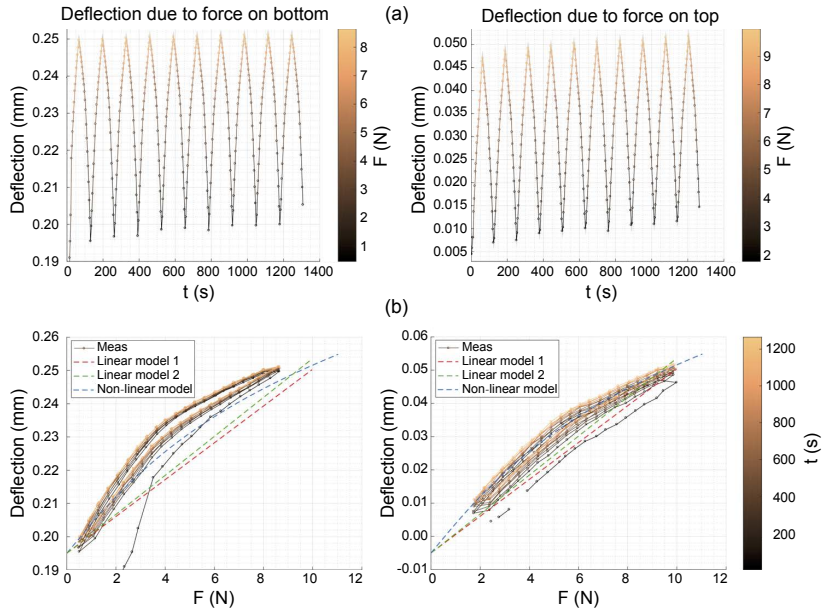

Figure 10. Repetitive mechanical loading of the strain gauges. Deflection versus time with colour coding for the load (a) and deflection versus load with colour-coded time (b) (Welleweerd, 2017).

black fractions would strongly increase resistivity and higher fractions would compromise printability. Combining polylactic acid (PLA) and the conductive PCL in a single print, a glove was printed in which tracks of the PCL embedded in the finger parts functioned as strain gauges. Relative resistance changes of a few percent were measured on bending the fingers of the glove. Other demonstrations were the printing of capacitive sensors as user input to electronic equipment
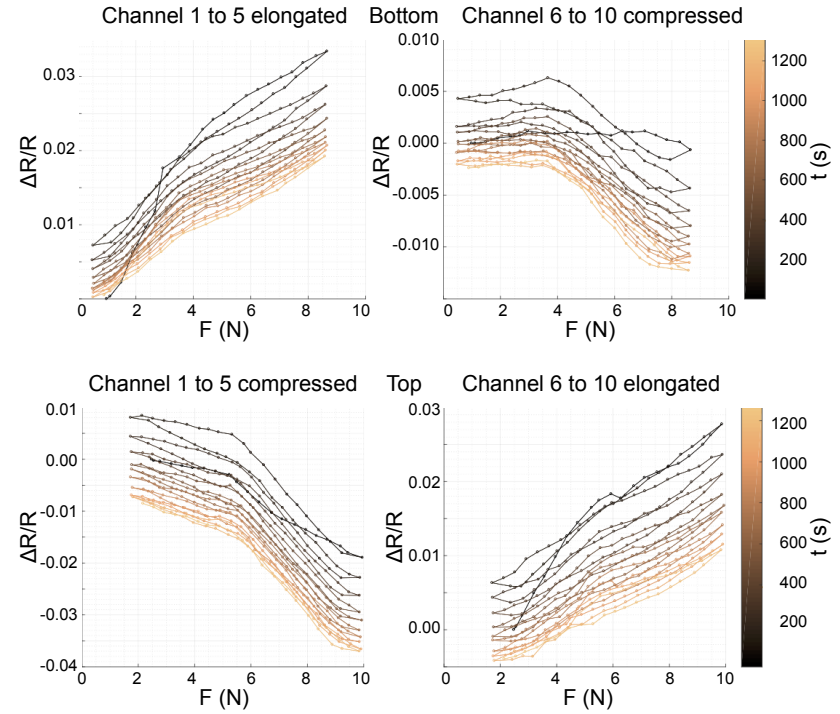

Figure 11. Repetitive mechanical loading of the strain gauges. Relative resistance change versus load with colour-coded time (Welleweerd, 2017).

and a smart mug where the water level in the mug was measured capacitively using the embedded electrodes.

Valentine et al. (2017) combined thermoplastic polyurethane (TPU) with AgTPU, i.e. TPU ink with added silver flakes. Printed AgTPU with up to $36 \%$ volume fraction $\mathrm{Ag}$ flakes yielded resistivities as low as $10^{-2} \Omega \mathrm{m}$. Since they contain the same solvent, the regular TPU and 


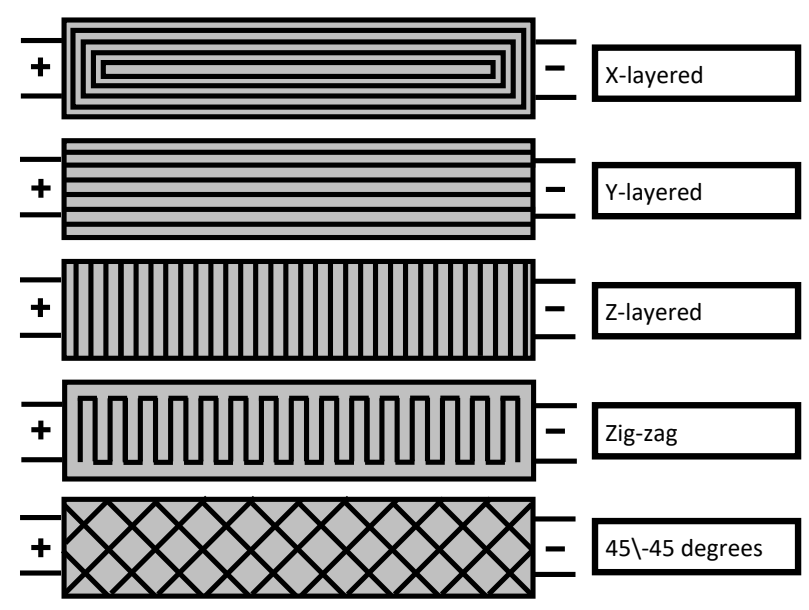

Figure 12. Different beam printing orientations for the resistivity experiments.

the conductive AgTPU combine well, causing a strong bond between layers of both types, preventing delamination, e.g. during stretching and flexing. The materials' combination was demonstrated on strain-sensor structures for the characterisation of elbow flexion and foot pressure measurements. Interestingly the necessary electronic components were integrated in the printed structures using a pick and place approach using an extra nozzle connected to a vacuum system to allow for automated assembly.

\subsection{Piezoresistive sensing experiments}

We investigated the possibilities of fabricating strain sensors consisting of co-printed dielectric beams and conductors.

\subsubsection{Conductive filament and properties}

Various conductive materials are commercially available for FDM printing. In this research we used Proto-pasta filament because of its high resisitivity (yielding an easy measurement set-up). The filament consists of PLA with carbon black filling as a conductive agent; see Proto-pasta (2016). Carbon black $(\mathrm{CB})$ is a term for various types of ultrafine paracrystalline carbon particles (10-400 nm diameter); see Fitz-Gerald and Boothe (2016). CB has a high heat resistance, high chemical resistance, a low mass density and low thermal expansion, and it provides electrical conductivity, which makes it suited as a filler in polymers. Several mechanisms may be involved in the conductivity of CB-filled conducting polymers, including quantum mechanical tunnelling, thermal expansion and mechanical interactions according to percolation theory (Huang, 2002). Proofs of concept for the application of the material in strain sensing research have, amongst others, focused on silicone (Muth et al., 2014), and PCL with CB (Leigh et al., 2012). Disadvantages of CBdoped polymers are their relatively high electrical resistance
Table 2. Resistivity for different printing orientations and infill patterns.

\begin{tabular}{lc}
\hline Orientation & Resistivity in $\Omega \mathrm{m}$ \\
\hline$X$-layered & 0.1090 \\
$Y$-layered & 0.0900 \\
$Z$-layered & 0.0911 \\
Zigzag & 0.2610 \\
$45-45^{\circ}$ & 0.1150 \\
\hline
\end{tabular}

(making them only suited for low-current applications) and their large creep deformation (Muller et al., 2015).

Proto-pasta filament is compatible with any PLA capable printer. The mechanical properties are comparable to undoped PLA, while the layer adhesion is worse (Proto-pasta, 2016). Due to anisotropy, the tensile strength and elastic properties of the part will vary depending on the built orientation (Ahn et al., 2002). The material shows increased brittleness relative to un-doped PLA.

\subsubsection{Experiments and results}

Experiments were done to determine both electrical and mechanical properties. Using four-wire measurements to eliminate contact resistances $(30 \Omega)$, the gauge resistances were determined by means of a source meter (Keithley 2440). Tests of the conductive properties showed that Proto-pasta is an ohmic material with a strong temperature-dependent resistivity. The resistivity could be determined for different printing orientations; see Fig. 12 and Table 2.

To determine the piezoresistive effect, a tensile test was performed with a universal tensile tester (Zwick, Model Z1.0) in combination with four-wire measurements. The change in resistance is plotted against the strain (Fig. 13). The correlation appears to be linear with exception of the interval $\left[0-3 \times 10^{-3}\right]$ (additional tests confirmed the linear behaviour for higher strains). The same non-linear behaviour at small $\varepsilon$ was found in literature for straining polymers with carbon nanoparticles (Levin et al., 2013). A possible explanation, as given in Cochrane et al. (2007), is the competition between structural changes due to the elongation and reorganisation of the conducting network.

From the obtained results a gauge factor can be derived, given by the ratio of relative change of resistance to mechanical strain. It is a combination of geometric terms and the piezoresistive effect. From the slope of the linear region in Fig. 13, the gauge factor of the material, $K=\frac{\Delta R / R_{0}}{\Delta \varepsilon}$, can be determined to be approximately 17.8. This gauge factor is much higher than what may be expected from geometric effects only, indicating a strong contribution from the piezoresistive effect of the material, and this gauge factor is high enough to provide usable results in various applications.

Finally, as a proof of concept, a planar and an integrated complex strain sensor were printed (Fig. 14). Resistances 


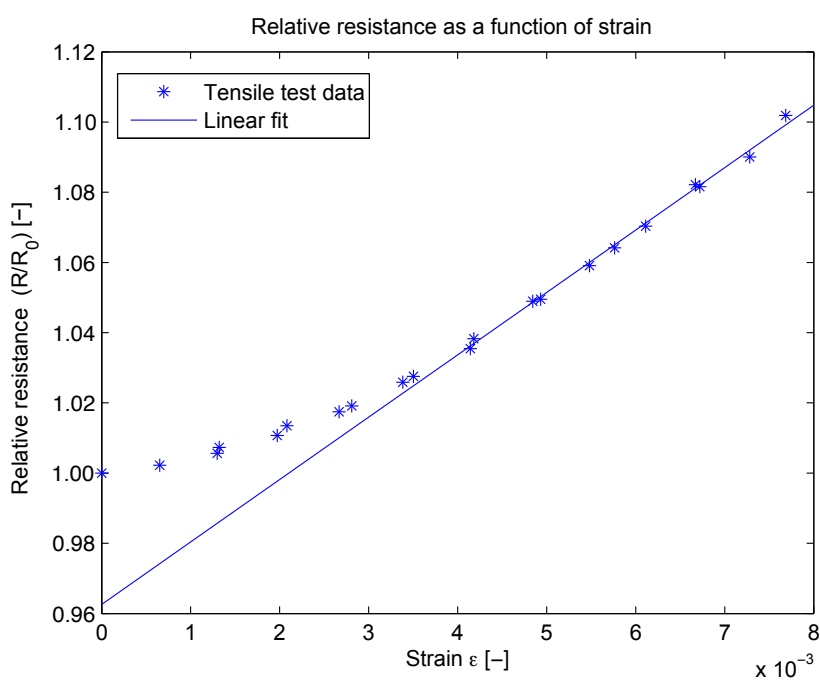

Figure 13. Resistance versus strain of tensile test.

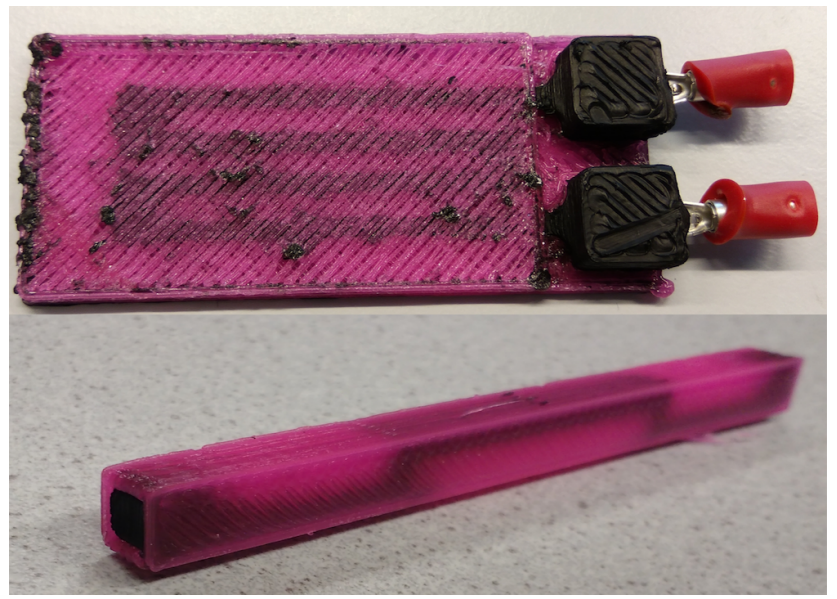

Figure 14. 3-D printed integrated strain sensors.

were measured and the functioning of the strain sensors were demonstrated qualitatively. From these experiments it can be concluded that Proto-pasta is suited for printing strain sensors. With additive manufacturing, integrated strain sensors with new advanced structures like in Fig. 14 are feasible.

\subsection{Co-printed whisker-inspired tactile sensors}

A follow-up of our work on tactile sensors used co-printed TPU structures, with piezoresistive sensors integrated in flexible beams which allow the structure to rotate and move sideways. A schematic picture of the structure can be seen in Fig. 15a.

For the whisker sensor two flexible materials are used. The first is NinjaFlex (Ninjatek); this flexible non-conductive material is used for the whisker and base of the design. The second material is a conductive TPU, PI-ETPU 95-250 Carbon Black (Palmiga Innovation). It is also based on carbon black

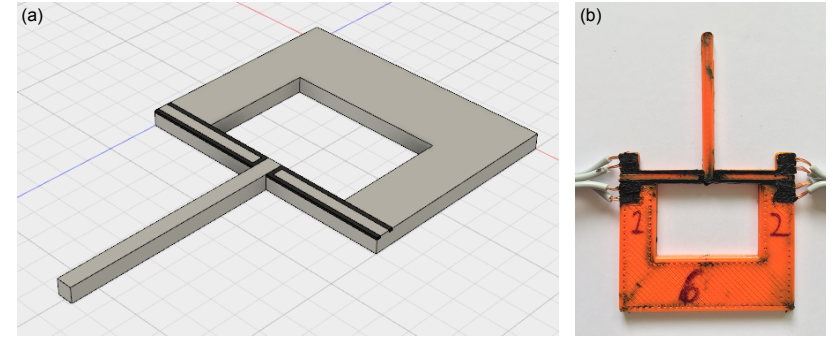

Figure 15. Panel (a) shows a CAD drawing of the co-printed tactile sensor. The black part is conductive. Panel (b) shows the printed version with molten connections (Eijking et al., 2017).

fillers, has a moderate resistivity $(<3 \Omega \mathrm{m})$ and can be 3-D printed. Both the dielectric NinjaFlex (Ninjatek) and conductive PI-ETPU (Palmiga Innovation) filaments are TPUbased and highly stretchable. Since these materials share the same base material, optimum printing conditions are highly comparable, allowing to co-print them in single parts with good adhesive properties. We have used a FlashForge Creator Pro (Flashforge) printer, modified with a direct drive Flexion extruder (Flexionextruder), essential for printing flexible materials.

It can be shown (Eijking et al., 2017) that the response of the sensors, based on suspension of the whisker on two flexible beams, can be written as

$\frac{\Delta R_{\mathrm{k}}}{R_{\mathrm{k}}}=\mathrm{GF} \times 2.86 \times 10^{-3}\left(\frac{L}{E I}\right)^{2} M^{2} \pm \frac{\mathrm{GF}}{A E} F_{\mathrm{ext}}$

with $k=\{\mathrm{L}, \mathrm{R}\}$,

where the subscripts $\mathrm{L}$ and $\mathrm{R}$ are used to indicate the left and right strain gauges respectively, $F_{\text {ext }}$ is the force acting on the whisker, $M=F_{\mathrm{ext}} \cdot S$ is the moment due to the force, $L$ is the length of each of the beams, $E$ is the Young's modulus of the material, $I$ is the second moment of area of the beams, $A$ is the area of the cross section of the beams and GF is the gauge factor. This expression shows that both force as well as moment, and hence the lever $(S)$ of the force relative to the rotation point, can be derived from the output of the two strain gauges (Eijking et al., 2017).

An example of a measurement is shown in Fig. 16. It clearly reflects the unsymmetrical response of both strain gauges due to the quadratic dependence on the applied moment (Eq. 1). The experimental curves were high-pass filtered to remove drift from the sensors' response. Additionally we observed hysteresis (Eijking et al., 2017, not shown here).

\subsection{FDM printed flexible surface electromyography (sEMG) sensors}

One of the exciting applications of embedded sensing is in the realm of soft robotics and medical applications such as 

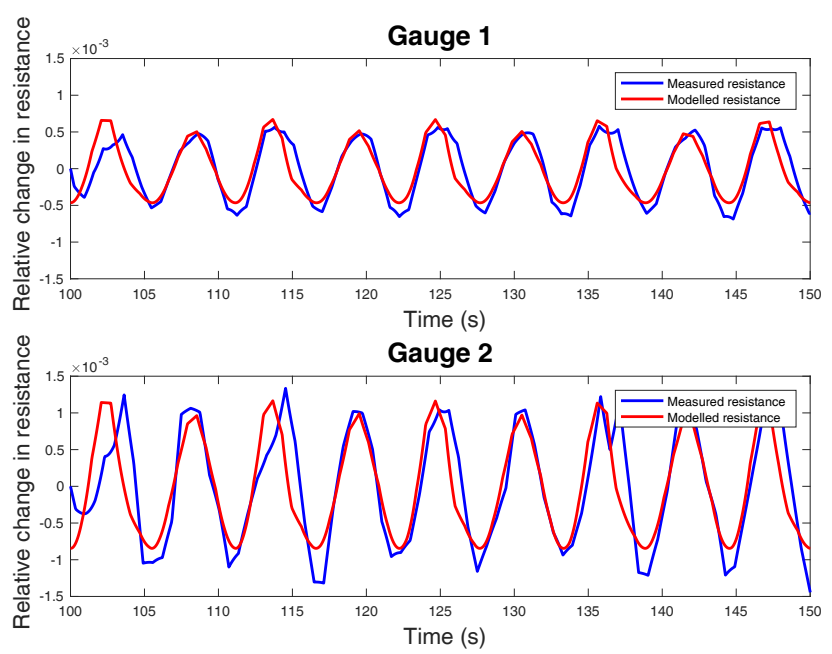

Figure 16. Comparison of measured (blue) and modelled (red) response of the two printed strain gauges after high-pass filtering (Eijking et al., 2017).

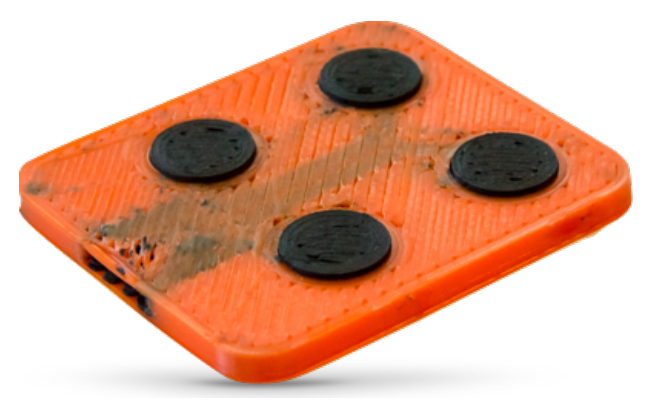

Figure 17. Photograph of 3-D printed sEMG electrodes, printed using a combination of conductive and dielectric TPU (Wolterink et al., 2017).

prosthesis and orthoses. These applications have in common that they contain soft structures which are difficult to sensorise traditionally. However, recent developments of both materials and printers have opened new possibilities for flexible prints, despite FDM being a relatively poor proposition for flexible materials compared to e.g. moulding and polyjetting. We used the same 3-D printer, direct extruders and materials as mentioned before for the whisker sensors.

In this study we are aiming for highly deformable surface electromyography (sEMG) sensors with the potential of making larger arrays and customised fits and integrating shielding, etc. An impression of such structures can be seen in Fig. 17 (Wolterink et al., 2017).

Utilising such structures, we tested the possibility of capturing muscle-activity-induced signals. The 3-D printed sensors were placed above the biceps brachii of a subject according to SENIAM recommendations (SENIAM), and we used a TMSi Refa amplifier to read, amplify and digitise the sEMG signals. Subsequently these were filtered $(5 \mathrm{~Hz}$ second-order high-pass filter and $50 \mathrm{~Hz}$ notch filter) using MatLab. First re-

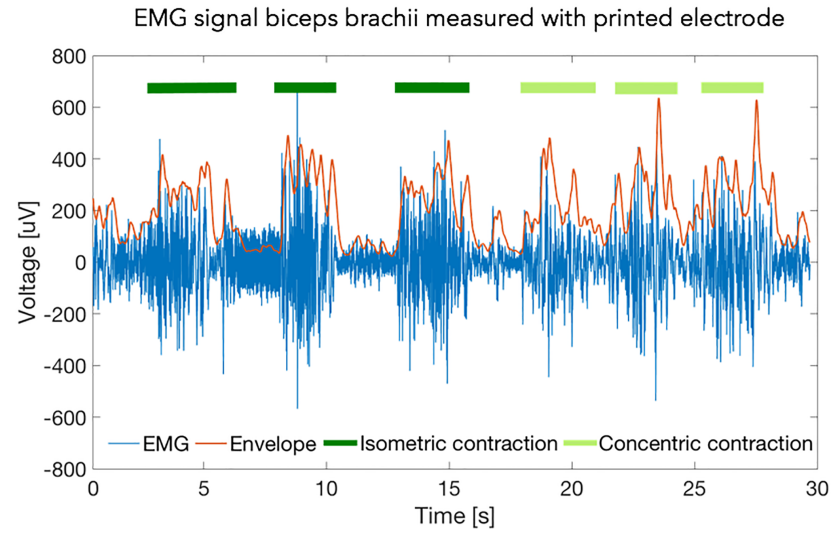

Figure 18. Electrical signals registered using a 3-D printed flexible sEMG sensor. Blue: raw signal after $5 \mathrm{~Hz}$ second-order highpass filtering and $50 \mathrm{~Hz}$ notch filtering. Red: envelope of the signal. Green bars indicate muscle activity.

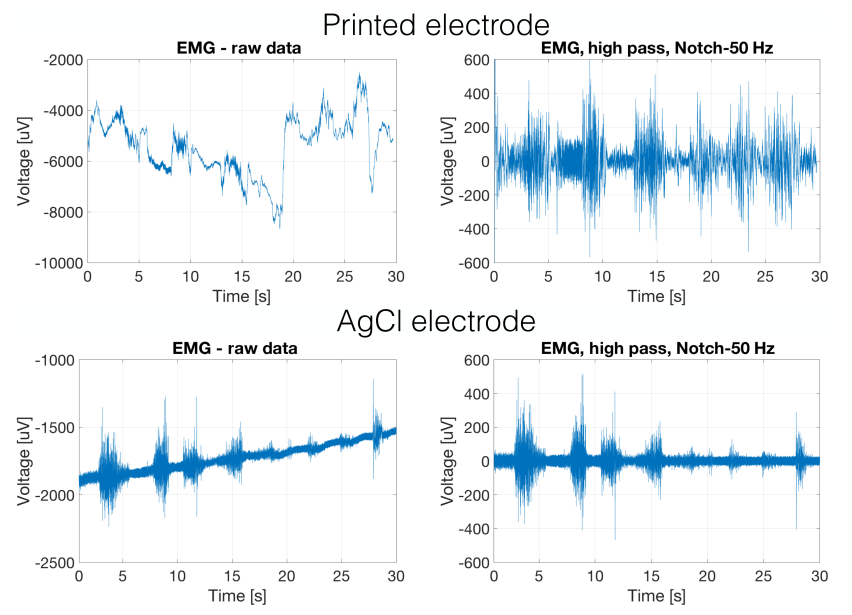

Figure 19. Comparison of sEMG signals acquired with printed and regular $\mathrm{AgCl}$ electrodes.

sults of such measurements are shown in Fig. 18 and indicate a high correlation between electrode signal and muscle activity. In comparison with regular $\mathrm{AgCl}$ electrodes (Fig. 19), we see that the printed sEMG signals are (a) in the same range, (b) have more noise and (c) have lower energy in the higher frequencies relative to the $\mathrm{AgCl}$ sensors (not shown here).

\section{Discussion}

In the examples given in this paper we have seen many non-idealities originating from mechanical sources, electrical sources or both. As long as plastics are involved, this may be something we have to expect. It would be straightforward to dismiss 3-D printed sensors for this reason. However, this would also mean that we miss out on the large potential of 3-D printed sensors, while adhering to the ideal engineering world, mainly consisting of linear systems. Nature tells 
us a different story; all sensing systems of the animal kingdom are based on non-linearities, as implied by the spiking properties of neural systems. And as we know, these systems perform extremely well. Hence, rather than abandoning 3D printed sensors, it becomes extremely interesting to think of systems which are resilient to the non-linearities of sensors, e.g. by smart signal processing (for example the effects of creep and drift can be alleviated by high-pass filtering) or by measurement and control strategies that show awareness of non-linearities. With the advent of 3-D printed sensors it will become increasingly easy to deploy many sensor(array)s. Nature forms a living example of what that may bring.

\section{Conclusions}

We have discussed various methods to make embedded sensors by 3-D printing and have given examples of these methods by sensors that have been presented in literature, as well as those developed in our lab-piezoresistive sensors (strain gauges), capacitive read-out and bio-electric sensing. A more or less common theme of these sensors seems to be their nonidealities in the form of non-linearities, creep and hysteresis. Since the potential of 3-D printed sensors is too large to be disregarded, our future research will be dedicated to innovative approaches to make ample use of these sensors.

Data availability. All relevant data presented in the article are stored according to institutional requirements and as such are not available online. However, all data used in this manuscript can be made available upon request to the authors.

Author contributions. AD, PW, MW, GW, BE and JD designed and fabricated the various 3-D printed devices, conducted the experiments and wrote corresponding text of the manuscript. RS conceived experiments and worked on gathering the experimental data. GK managed the research in this manuscript, conceived and wrote large parts of the manuscript and contributed to the modelling.

Competing interests. The authors declare that they have no personal, financial or other ties to the manufacturers of equipment or materials discussed in this paper and neither did they receive any financial or other benefits or privileges from such manufacturers. Therefore this manuscript is an unbiased account of their experimental findings, insights, models and sources available in the public domain.

Special issue statement. This article is part of the special issue "Sensor/IRS2 2017". It is a result of the AMA Conferences, Nuremberg, Germany, 30 May-1 June 2017.
Acknowledgements. The research leading to these results has received funding from the European Union's Horizon 2020 Research and Innovation Programme under grant agreement no. 688857 (SoftPro).

Edited by: Stefan Rupitsch

Reviewed by: one anonymous referee

\section{References}

Ahn, S.-H., Montero, M., Odell, D., Roundy, S., and Wright, P. K.: Anisotropic material properties of fused deposition modeling ABS, Rapid Prototyping J., 8, 248-257, 2002.

Boland, C. S., Khan, U., Backes, C., Neill, A. O., Mccauley, J., Duane, S., Shanker, R., Liu, Y., Jurewicz, I., Dalton, A. B., and Coleman, J. N.: Sensitive, High-Strain, High-Rate Bodily Motion Sensors Based on Graphene-Rubber Composites, 8, 88198830, https://doi.org/10.1021/nn503454h, 2014.

Borghetti, M., Serpelloni, M., Sardini, E., and Pandini, S.: Mechanical behavior of strain sensors based on PEDOT:PSS and silver nanoparticles inks deposited on polymer substrate by inkjet printing, Sensor. Actuat. A Phys., 243, 71-80, https://doi.org/10.1016/j.sna.2016.03.021, 2016.

Cochrane, C., Koncar, V., Lewandowski, M., and Dufour, C.: Design and development of a flexible strain sensor for textile structures based on a conductive polymer composite, Sensors, 7, 473492, 2007.

Czyzewski, J., Burzyński, P., Gaweł, K., and Meisner, J.: Rapid prototyping of electrically conductive components using 3D printing technology, J. Material. Process. Technol., 209, 5281-5285, https://doi.org/10.1016/j.jmatprotec.2009.03.015, 2009.

Delamare, J., Sanders, R., and Krijnen, G.: 3D printed biomimetic whisker-based sensor with co-planar capacitive sensing, in: 2016 IEEE SENSORS, 1-3, https://doi.org/10.1109/ICSENS.2016.7808631, 2016.

Eijking, B., Sanders, R., and Krijnen, G.: Development of Whisker Inspired 3D Multi-Material Printed Flexible Tactile Sensors, in: IEEE Sensors 2017, IEEE, IEEE, Piscataway, https://doi.org/10.1109/ICSENS.2017.8233952, 2017.

Fitz-Gerald, D. and Boothe, J.: Manufacturing and Characterization of Poly (Lactic Acid)/Carbon Black Conductive Composites for FDM Feedstock: An Exploratory Study, available at: http://digitalcommons.calpoly.edu/matesp/152/ (last access: 16 February 2018), 2016.

Flashforge: Creator Pro 3D printer, available at: http://www. flashforge.com/creator-pro-3d-printer/, last access: 16 February, 2018.

Flexionextruder: Dual Flexion retrofit kit, available at: https:// flexionextruder.com/shop/dual/, last access: 16 February, 2018.

Habas, S. E., Platt, H. A. S., Van Hest, M. F. A. M., and Ginley, D. S.: Low-cost inorganic solar cells: From ink to printed device, Chem. Rev., 110, 6571-6594, https://doi.org/10.1021/cr100191d, 2010.

Harada, S., Kanao, K., Yamamoto, Y., Arie, T., Akita, S., and Takei, K.: Fully Printed Flexible Fingerprint-like Three-Axis Tactile and Slip Force and Temperature Sensors for Arti fi cial Skin, ACS Nano, 8, 12 851-12 857, 2014. 
Huang, J.-C.: Carbon black filled conducting polymers and polymer blends, Adv. Polymer Technol., 21, 299-313, https://doi.org/10.1002/adv.10025, 2002.

Kirkpatrick, M. B., Tarbutton, J. A., Le, T., and Lee, C.: Characterization of 3D printed piezoelectric sensors: Determiniation of $\mathrm{d} 33$ piezoelectric coefficient for 3D printed polyvinylidene fluoride sensors, in: 2016 IEEE SENSORS, 1-3, https://doi.org/10.1109/ICSENS.2016.7808876, 2016.

Ko, S. H., Chung, J., Hotz, N., Nam, K. H., and Grigoropoulos, C. P.: Metal nanoparticle direct inkjet printing for low-temperature 3D micro metal structure fabrication, J. Micromechan. Microeng., 20, 125010, https://doi.org/10.1088/0960-1317/20/12/125010, 2010.

Krijnen, G. J. and Sanders, R. G.: Recent Developments in Bio-Inspired Sensors Fabricated by Additive Manufacturing Technologies, Adv. Sci. Technol., 100, 197-206, https://doi.org/10.4028/www.scientific.net/AST.100.197, 2016.

Leigh, S. J., Bradley, R. J., Purssell, C. P., Billson, D. R., and Hutchins, D. A.: A Simple, Low-Cost Conductive Composite Material for 3D Printing of Electronic Sensors, PLoS ONE, 7, 1-6, https://doi.org/10.1371/journal.pone.0049365, 2012.

Leigh, S. J., Purssell, C. P., Billson, D. R., and Hutchins, D. A.: Using a magnetite/thermoplastic composite in $3 \mathrm{D}$ printing of direct replacements for commercially available flow sensors Abstract - Smart Materials and Structures - IOPscience, Smart Material. Struct., 23, 095039, https://doi.org/10.1088/09641726/23/9/095039, 2014.

Levin, Z., Robert, C., Feller, J., Castro, M., and Grunlan, J.: Flexible latex - Polyaniline segregated network composite coating capable of measuring large strain on epoxy, Smart Material. Struct., 22, 015008, https://doi.org/10.1088/09641726/22/1/015008, 2013.

Liu, Y., Cui, T., and Varahramyan, K.: All-polymer capacitor fabricated with inkjet printing technique, Solid-State Electron., 47, 1543-1548, https://doi.org/10.1016/S0038-1101(03)00082$0,2003$.

Lopes, A. J., Lee, I. H., MacDonald, E., Quintana, R., and Wicker, R.: Laser curing of silver-based conductive inks for in situ 3D structural electronics fabrication in stereolithography, J. Material. Process. Technol., 214, 1935-1945, https://doi.org/10.1016/j.jmatprotec.2014.04.009, 2014.

MacDonald, E., Salas, R., Espalin, D., Perez, M., Aguilera, E., Muse, D., and Wicker, R. B.: 3D printing for the rapid prototyping of structural electronics, IEEE Access, 2, 234-242, https://doi.org/10.1109/ACCESS.2014.2311810, 2014.

Muller, V., Fritzsche, M., and Elkmann, N.: Sensor design and calibration of piezoresistive composite material, IEEE Sensors, Busan, 2015, 1-4, https://doi.org/10.1109/ICSENS.2015.7370488, 2015.

Muth, J. T., Vogt, D. M., Truby, R. L., Meng, Y., Kolesky, D. B., Wood, R. J., and Lewis, J. A.: Embedded 3D printing of strain sensors within highly stretchable elastomers, Adva. Material., 26, 6307-6312, https://doi.org/10.1002/adma.201400334, 2014.

Ninjatek: NinjaFlex flexible filament, available at: https://ninjatek. com/products/filaments/ninjaflex/, last access: 16 February, 2018.

OpenScad: The Programmers Solid 3D CAD Modeller, available at: http://www.openscad.org, last access: 16 February, 2018.
ORD: RoVa3D printers, available at: http://www.ordsolutions.com/ rova3d/, last access: 16 February, 2018.

Ota, H., Emaminejad, S., Gao, Y., Zhao, A., Wu, E., Challa, S., Chen, K., Fahad, H. M., Jha, A. K., Kiriya, D., Gao, W., Shiraki, H., Morioka, K., Ferguson, A. R., Healy, K. E., Davis, R. W., and Javey, A.: Application of 3D Printing for Smart Objects with Embedded Electronic Sensors and Systems, Adv. Mater. Technol., 1, 1-8, https://doi.org/10.1002/admt.201600013, 2016.

Palmiga Innovation: Material info for PI-ETPU 95-250 Carbon Black the conductive and flexible 3D printing filament, available at: http://rubber3dprinting.com/pi-etpu-95-250-carbon-black/, last access: 16 February, 2018.

Pella, T.: Conductive carbon glue, Web, available at: https:// www.tedpella.com/technote_html/TN_16050.pdf, last access: 16 February, 2018.

Proto-pasta: Conductive PLA, available at: https://www. proto-pasta.com/pages/conductive-pla (last access: 16 February 2018) 2016.

Rajala, S. N. K., Mettänen, M., and Tuukkanen, S.: Structural and Electrical Characterization of Solution-Processed Electrodes for Piezoelectric Polymer Film Sensors, IEEE Sensors J., 16, 16921699, https://doi.org/10.1109/JSEN.2015.2504956, 2016.

SENIAM: Seniam website, available at: http://seniam.org, last access: 16 February, 2018.

Senturia, S. D.: Microsystem Design, kluwer Academic Publishers, New York, Boston, Dordrecht, London, Moscow, 2001.

Shemelya, C., Banuelos-Chacon, L., Melendez, A., Kief, C., Espalin, D., Wicker, R., Krijnen, G., and Macdonald, E.: Multifunctional 3D printed and embedded sensors for satellite qualification structures, 2015 IEEE SENSORS - Proceedings, 1-4, https://doi.org/10.1109/ICSENS.2015.7370541, 2015a.

Shemelya, C., Cedillos, F., Aguilera, E., Espalin, D., Muse, D., Wicker, R., and Macdonald, E.: Encapsulated copper wire and copper mesh capacitive sensing for 3-D printing applications, IEEE Sensors J., 15, 1280-1286, https://doi.org/10.1109/JSEN.2014.2356973, 2015b.

Song, H., Zhang, J., Chen, D., Wang, K., Niu, S., Han, Z., and Ren, L.: Superfast and high-sensitivity printable strain sensors with bioinspired micron-scale cracks, Nanoscale, 9, 1166, https://doi.org/10.1039/C6NR07333F, 2017.

Stratasys: Connex3D printers, available at: http://www.stratasys. com/3d-printers/production-series/connex3-systems, last access: 16 February, 2018.

Valentine, A. D., Busbee, T. A., Boley, J. W., Raney, J. R., Chortos, A., Kotikian, A., Berrigan, J. D., Durstock, M. F., and Lewis, J. A.: Hybrid 3D Printing of Soft Electronics, Adv. Mater., 29, 1-8, https://doi.org/10.1002/adma.201703817, 2017.

van Tiem, J., Groenesteijn, J., Sanders, R., and Krijnen, G.: 3D printed bio-inspired angular acceleration sensor, in: 2015 IEEE SENSORS, 1-4, https://doi.org/10.1109/ICSENS.2015.7370543, 2015.

Vatani, M., Engeberg, E. D., and Choi, J.-W.: Conformal directprint of piezoresistive polymer/nanocomposites for compliant multi-layer tactile sensors, Additive Manufact., 7, 73-82, https://doi.org/10.1016/j.addma.2014.12.009, 2015.

Voxel8: Multi-Material Digital Manufacturing, available at: http:// www.voxel8.com, last access: 16 February, 2018.

Welleweerd, M.: 3D printing a three degree of freedom force sensor, Master's thesis, University of Twente, EE dept., 2017. 
Wolterink, G., Sanders, R., Muijzer, F., van Beijnum, B.-J., and Krijnen, G.: 3D-Printing Soft sEMG Sensing Structures, in: IEEE Sensors 2017, IEEE, IEEE, Piscataway, https://doi.org/10.1109/ICSENS.2017.8233935, 2017.

Wu, S.-Y., Yang, C., Hsu, W., and Lin, L.: 3D-printed microelectronics for integrated circuitry and passive wireless sensors, Microsyst. Nanoeng., 1, 15013, https://doi.org/10.1038/micronano.2015.13, 2015.

Zhao, J., Dai, K., Liu, C., Zheng, G., Wang, B., Liu, C., Chen, J., and Shen, C.: A comparison between strain sensing behaviors of carbon black/polypropylene and carbon nanotubes/polypropylene electrically conductive composites, Compos. Part A Appl. S., 48, 129-136, https://doi.org/10.1016/j.compositesa.2013.01.004, 2013.
Zin, R. M., Soon, C. F., Ghadafi, S., Ali, R. A. M., and Nayan, N.: Fabrication and characterisation of the electrical and physical properties of the mask printed graphite paste electrodes on paper substrates, Int. J. Nanoelectr. Material., 8, 47-53, https://doi.org/10.4028/www.scientific.net/AMR.925.510, 2015. 